\title{
Mineralocorticoid receptor blockers for moderate kidney dysfunction: more merit than ever?
}

\author{
Masashi Mukoyama ${ }^{1} \cdot$ Takashige Kuwabara $^{1} \cdot$ Masataka Adachi $^{1}$
}

Received: 28 May 2021 / Accepted: 31 May 2021 / Published online: 15 July 2021

(c) The Japanese Society of Hypertension 2021

Mineralocorticoid receptor (MR) antagonists, such as spironolactone and eplerenone, are currently used to treat hypertension and chronic heart failure. MR antagonists act on MRs expressed in the distal tubules of the kidney, including distal convoluted tubules, connecting tubules and the cortical collecting duct, thereby promoting sodium excretion without the loss of potassium into the urine [1]. MR antagonists may be effective for the treatment of lowrenin hypertension and are preferentially used for the treatment of primary aldosteronism; furthermore, in patients with resistant hypertension, additional administration at a low to moderate dose (spironolactone, $25-50 \mathrm{mg} /$ day) may further decrease blood pressure [2].

Accumulating evidence has shown that aldosterone can act outside the renal tubules, leading to adverse effects, such as oxidative stress, endothelial dysfunction, inflammation, and fibrosis, especially within the heart, vasculature, and kidney glomerulus/interstitium. In this regard, a number of studies have been conducted on MR antagonists to verify their organprotecting effects in patients with heart failure or those with chronic kidney disease (CKD) [2]. In fact, spironolactone and eplerenone were revealed to be cardioprotective and also to reduce proteinuria $[2,3]$. These agents, however, may result in adverse events such as hyperkalemia and worsening of renal function, particularly when given in addition to reninangiotensin system (RAS) inhibitors in patients with CKD [2]. Furthermore, spironolactone could cause gynecomastia and impotence [2] because of its relatively low specificity for the MR.

To overcome the disadvantages of MR antagonists, novel, selective nonsteroidal agents have been developed. Esaxerenone is a potent, nonsteroidal oral MR blocker that inhibits

Masashi Mukoyama

mmuko@kumamoto-u.ac.jp

1 Department of Nephrology, Kumamoto University Graduate School of Medical Sciences, Kumamoto, Japan
MR activity with high specificity. To date, several clinical studies have been performed to evaluate its effects [4-6], which revealed effective blood pressure reduction with relatively few adverse events, including hyperkalemia (Table 1). In addition, esaxerenone can further reduce urinary albumin excretion in addition to RAS inhibitors without significantly affecting renal function [6]. Finerenone, a second nonsteroidal MR blocker for which large-scale clinical trials have been completed [7, 8], showed a lower risk of CKD progression and cardiovascular events than placebo in patients with CKD and type 2 diabetes (Table 1). Based on these results, much attention has been given to novel MR blockers as promising organ-protective agents for hypertensive patients with CKD.

In the latest issue of Hypertension Research, Ito et al. reported the results of esaxerenone administration in 91 hypertensive patients ( 33 with monotherapy and 58 with addon therapy) with moderate kidney dysfunction (estimated glomerular filtration rate (eGFR) $\geq 30$ and $<60 \mathrm{~mL} / \mathrm{min} /$ $1.73 \mathrm{~m}^{2}$ ) for 12 weeks, which revealed a significant blood pressure reduction and stabilization of renal function [9]. Reductions in sitting blood pressure from baseline were similar in both the monotherapy and add-on therapy groups (by 17-18/8-9 mmHg) (Table 1). A mild transient increase in serum potassium levels was observed in 7 patients receiving add-on therapy but not in the patients receiving monotherapy, with no one discontinuing therapy. Although this is a singlearm study, the results indicate that esaxerenone may be more beneficial than previously considered based on previous studies using spironolactone or eplerenone.

MR-related organ damage, leading to cardiovascular disease and CKD progression, has been increasingly recognized (Fig. 1). Such pathological conditions could be brought about through not only aldosterone-dependent but also aldosterone-independent pathways [10], including obesity, diabetes, excessive salt intake, and some forms of CKD. The current study suggests that esaxerenone is useful to treat hypertension in patients with CKD and can also prevent organ damage, perhaps irrespective of aldosterone dependence. Although attention should be given to the 


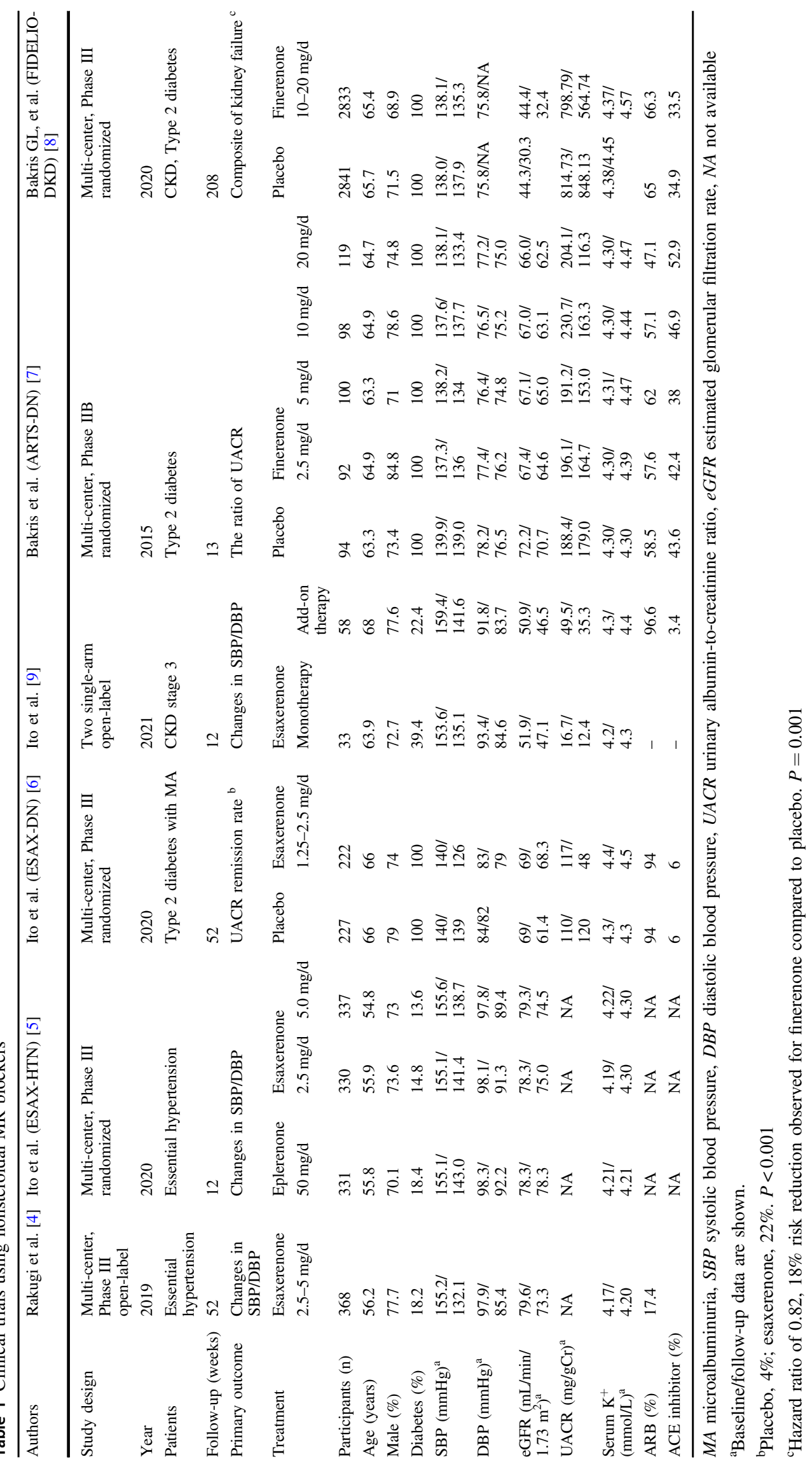


Fig. 1 Proposed actions of esaxerenone in MR-related organ damage.

Mineralocorticoid receptor

(MR)-related organ damage can occur in both aldosteronedependent and aldosteroneindependent pathways, eventually leading to cardiovascular disease (CVD) and CKD progression.

Esaxerenone, a nonsteroidal MR blocker, is useful for treating hypertension in patients with CKD and is likely to reduce the risk of organ damage, although attention should be given to the possible occurrence of hyperkalemia and metabolic acidosis. RAS, renin-angiotensin system; $11 \beta$-HSD2,

11ß-hydroxysteroid dehydrogenase type 2
Risk factors for MR-related organ damage

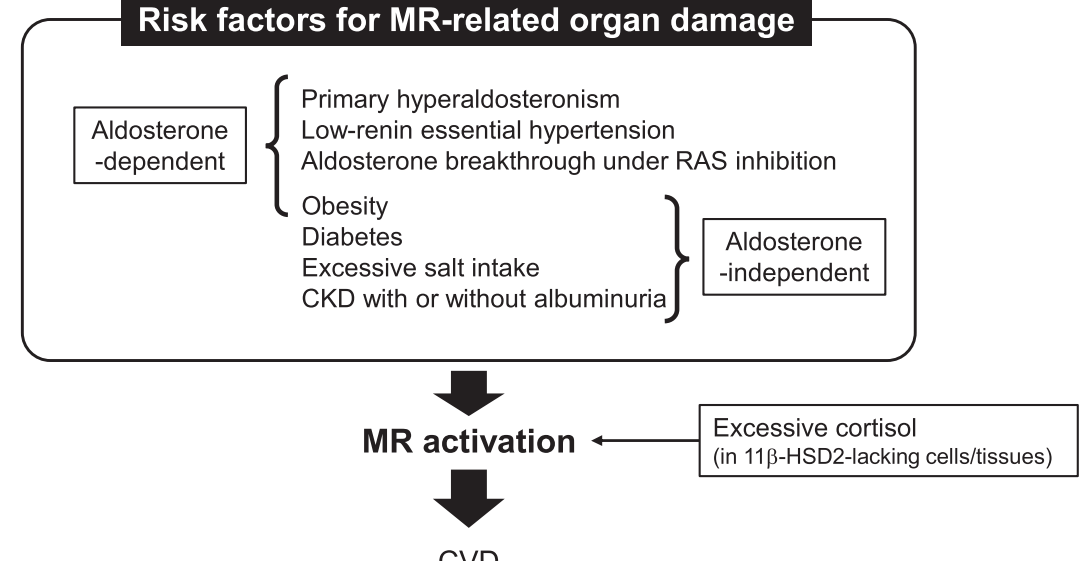

CVD

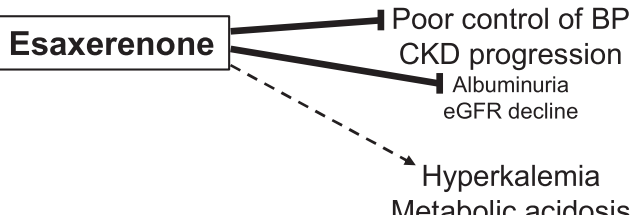

possible occurrence of hyperkalemia and metabolic acidosis when using esaxerenone in patients with CKD, further investigation and studies with hard endpoints in a larger number of patients should be encouraged to verify advantages for its use in patients with hypertension and CKD.

\section{Compliance with ethical standards}

Conflict of interest The authors declare no competing interests.

Publisher's note Springer Nature remains neutral with regard to jurisdictional claims in published maps and institutional affiliations.

\section{References}

1. Shibata S. 30 YEARS OF THE MINERALOCORTICOID RECEPTOR: mineralocorticoid receptor and $\mathrm{NaCl}$ transport mechanisms in the renal distal nephron. J Endocrinol. 2017;234: T35-47.

2. Umemura S, Arima H, Arima S, Asayama K, Dohi Y, Hirooka Y, et al. The Japanese Society of Hypertension guidelines for the management of hypertension (JSH2019). Hypertens Res. 2019;42: 1235-481.

3. Nishimoto M, Ohtsu H, Marumo T, Kawarazaki W, Ayuzawa N, Ueda K, et al. Mineralocorticoid receptor blockade suppresses dietary salt-induced ACEI/ARB-resistant albuminuria in non-diabetic hypertension: a sub-analysis of evaluate study. Hypertens Res. 2019;42:514-21.

4. Rakugi H, Ito S, Itoh $\mathrm{H}$, Okuda Y, Yamakawa S. Long-term phase 3 study of esaxerenone as mono or combination therapy with other antihypertensive drugs in patients with essential hypertension. Hypertens Res. 2019;42:1932-41.

5. Ito S, Itoh H, Rakugi H, Okuda Y, Yoshimura M, Yamakawa S. Double-blind randomized phase 3 study comparing esaxerenone (CS-3150) and eplerenone in patients with essential hypertension (ESAX-HTN Study). Hypertension. 2020;75:51-8.

6. Ito S, Kashihara N, Shikata K, Nangaku M, Wada T, Okuda Y, et al. Esaxerenone (CS-3150) in patients with type 2 diabetes and microalbuminuria (ESAX-DN): Phase 3 randomized controlled clinical trial. Clin J Am Soc Nephrol 2020;15:1715-27.

7. Bakris GL, Agarwal R, Chan JC, Cooper ME, Gansevoort RT, Haller $\mathrm{H}$, et al. Mineralocorticoid Receptor Antagonist Tolerability Study-Diabetic Nephropathy (ARTS-DN) Study Group. Effect of finerenone on albuminuria in patients with diabetic nephropathy: a randomized clinical trial. JAMA. 2015; 314:884-94.

8. Bakris GL, Agarwal R, Anker SD, Pitt B, Ruilope LM, Rossing P, et al. FIDELIO-DKD Investigators. Effect of finerenone on chronic kidney disease outcomes in type 2 diabetes. N Engl J Med. 2020;383:2219-29.

9. Ito S, Itoh H, Rakugi H, Okuda Y, Iijima S. Antihypertensive effects and safety of esaxerenone in patients with moderate kidney dysfunction. Hypertens Res. 2021;44:489-97.

10. Ayuzawa N, Fujita T. The mineralocorticoid receptor in saltsensitive hypertension and renal injury. J Am Soc Nephrol. 2021; $32: 279-89$. 